\title{
Flexible testing without programming
}

\author{
DOUGLAS M. KLIEGER \\ Villanova University, Villanova, Pennsylvania
}

\begin{abstract}
Test administration by computer has been available for some time, yet such procedures have been little more than electronic substitutes for conventional paper-and-pencil tests. The Flexible Tester allows the researcher to ask as many subquestions as desired and make all questions contingent upon subject response. The logic of questioning is created through a dialogue with the program rather than by direct programming. The program is suitable for any questioning procedure, including structured interviews expressible in the false/true format.
\end{abstract}

The term adaptive testing as it is used in the literature is limited. Weiss (1985) defines an adaptive test as "one in which different sets of test questions (items) are administered to different individuals depending on each individual's status on the trait being measured"' (p. 774). In this way, questions irrelevant to the individual are skipped, and the process is more efficient in general. These procedures are tied to item response theory (Weiss, 1985), and most often they are applied in ability testing. However, the extensive developmental work in this area has not led to general-purpose procedures, despite the power and flexibility inherent in "computerized adaptive testing" (Weiss, 1985). My purpose in this paper is to define and operationalize a form of adaptive testing not tied to a particular theory, or to particular goals-an adaptive test in the generic sense.

The program that follows pursues a number of traditional goals in computer-administered testing. It is branched if, and only if, the researcher wishes it so. The program is sequential if that is the desired goal, and almost any combination of sequential and/or branching operation is possible. The program is response-contingent in that it can respond differentially. Finally, the program is not confined to ability testing. The only requirements are a false/true format and a clear notion on the part of the researcher of what questions ought to follow specific responses. Thus the program is similar to, and indeed adaptable to, the structured interview.

For example, Klieger $(1987,1989)$ has demonstrated that the Snake Anxiety Questionnaire (SNAQ; Klorman, Weerts, Hastings, Melamed, \& Lang, 1974) is subject to a high false positive rate; that is, it identifies as fearful of snakes many individuals for whom no other evidence of fear can be found. If subjects who obtain high SNAQ scores are interviewed about their ostensible fear, several points emerge. The response "true" to the question "I would feel uncomfortable wearing a snakeskin belt" may indicate a philosophical position about the destruction of animals rather than any fear. The question "I avoid go-

Correspondence should be addressed to Douglas M. Klieger, Department of Psychology, Villanova University, Villanova, PA 19085. ing to parks or on camping trips because there may be snakes about" virtually demands follow-up questions about poisonous/nonpoisonous snakes, dislike of all outdoor activities, avoidance because a close relation is fearful of snakes, and so forth. Of course, most testing procedures attempt to compensate for the lack of item precision by asking many questions. If all the items are imprecise, hundreds of questions and thousands of subjects may be required to produce a reliable and valid procedure. It is more efficient, and certainly more direct, to simply ask the detailed follow-up questions.

The Flexible Tester is a group of procedures for administering false/true questionnaires of any length, followed by any number of follow-up questions. The Flexible Tester supports two principle testing variations: one, all main (original) questions followed by subquestions with or without branching; and two, main questions immediately followed by the relevant subquestions. Thus, for the SNAQ, the researcher would clearly wish to have the subject respond to the questionnaire in its entirety before proceeding to follow-up questions. The SNAQ with interposed subquestions would no longer be the SNAQ. On the other hand, the Fear Survey Schedule (FSS; Wolpe \& Lang, 1964) does not have established scale scores, and thus intervening subquestions cannot interfere. Each question on the FSS is virtually a one-item test. The Flexible Tester can accommodate both approaches.

Branching in the Flexible Tester does not depend on the ability of the individual researcher to construct Boolean logic in the form of a programming language. The approach utilized by the Flexible Tester is to hold a dialogue with the user. For example, FSS Question 47 (Wolpe \& Lang, 1977) asks respondents to rate their fear of fire. Assuming that a rating of "very much" is the equivalent of saying "true, I am afraid of fire," several follow-up questions would appear essential, because preliminary validity work by this author has indicated that subjects interpret this question rather broadly. Answering based on recent movie and television dramas is common, as is envisioning personal harm from fire. These individuals have no difficulty approaching laboratory fires. The Flexible Tester allows the researcher not only to ask logical follow-up questions such as "Have you ever 
been forced to flee from a burning building/house?' but to branch to further questions, depending on the answers. In this example, the researcher might wish to terminate this line of questions if the subject responds "no," yet ask about "personal harm" if the subject responds "yes."

The Flexible Tester avoids involving the researcher in elaborate IF statements, DO loops, and similar constructs common to most programming languages. The sequence of questions for the example above is constructed simply by asking the researcher what should follow what. To achieve a "no programming" environment, the Flexible Tester asks the researcher for the identification number of the question/subquestion that is to follow a false response, then true and so on. Figure 1 details an elaborate example for the SNAQ question, "If a picture of a snake appears on the screen during a motion picture, I turn my head away." This question virtually demands follow-up questions about the behavior, for it relates to other classic fear stimuli, watching films not manipulated to be frightening, and probing for evidence of irrational- ity. The logic presented in Figure 1 is not presented as the only solution to the lack of precision in the original SNAQ question; it is rather an example of the elaborations that are possible.

The Flexible Tester can administer follow-up sequences, with the restriction that only false/true responding is allowed. This is not as restrictive as it appears, in that most lines of questioning can be couched in twovalued logic. Also, there is no restriction on repeating the same question. Thus, the testing procedure can attain a more involved logic through the device of allowing the same question to appear in multiple logic chains. The advisability of presenting the same question to the subject is a separate issue.

Scoring of questionnaires such as the SNAQ is provided for, but there is no provision for scoring of follow-up questions. Indeed, the logic of scoring subquestions is dubious from the perspective of a general-purpose procedure. However, subject responses are stored in files that are easily readable and are printed as well. The Flexible

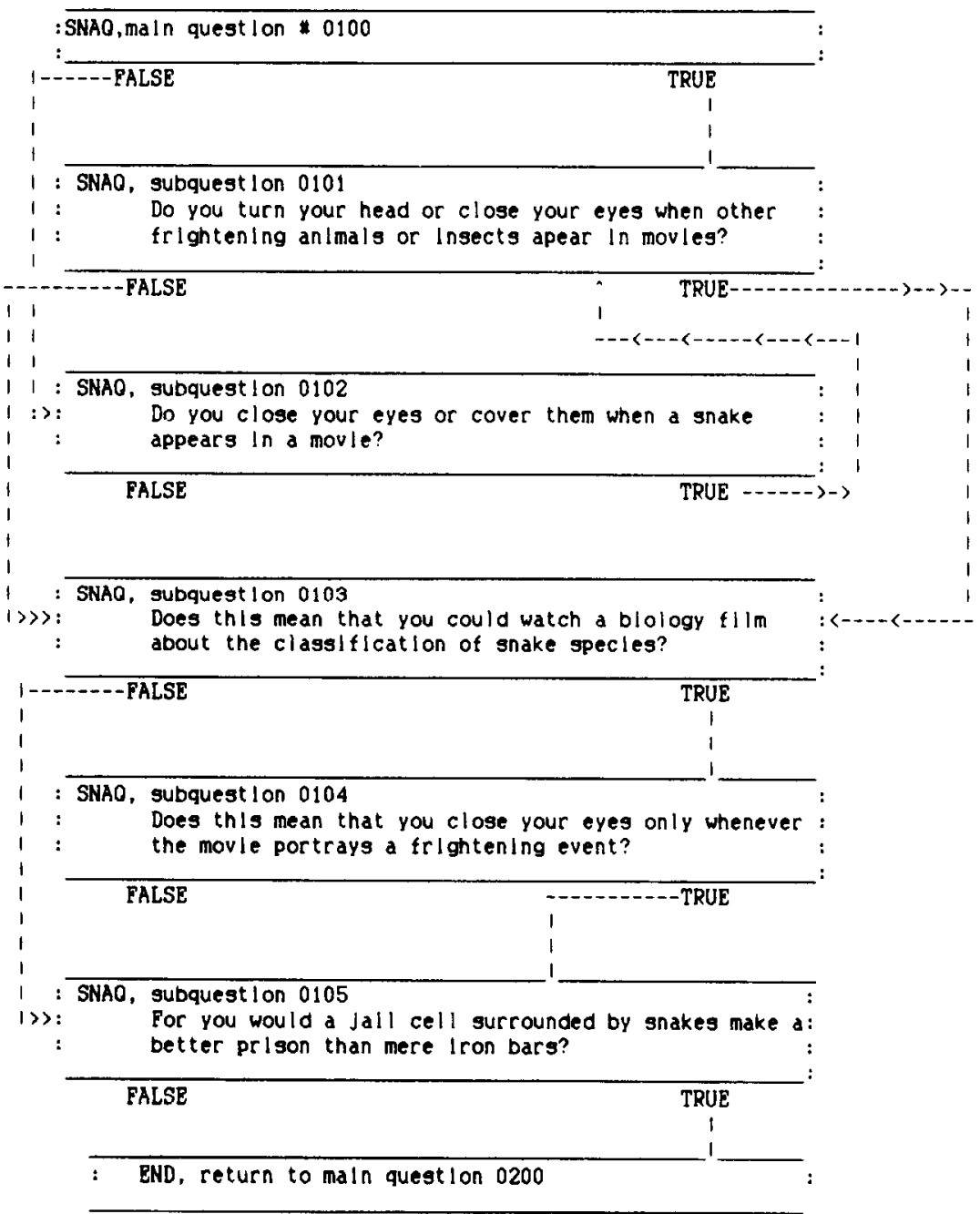

Figure 1. A block diagram of hypothetical SNAQ subquestion logic. 
Tester can be modified to accommodate custom scoring by any user familiar with the BASIC programming language and PRODOS operating system. All output files are written in a form accessible to any program that can read PRODOS files.

\section{Program}

The Flexible Tester is written in TML BASIC (TML Systems, 1987), which is a compiled language rather than the typical interpreted BASIC. This variant provides a number of important advantages; line numbers are not required, for example, nor are there the typical restrictions on variable names. Programs are far more readable when variable names may be spelled out in full without subsequent performance penalties. Thus, in the Flexible Tester, subject responses become MAINRESPONSE\% and ANSWER\$, with the \% and \$ symbols denoting integer and string variables, respectively. TML BASIC also facilitates the construction of procedures, functions, and libraries, which in turn promote a structured approach to programming. A compiled program does not require the user to possess the programming language. Compiled programs are true stand-alone programs.

The Flexible Tester takes a modular approach to program construction through the device of defining and calling procedures. Thus, there are procedures for defining main questions, subquestions, scoring, housekeeping, and so forth. This approach creates a more readable program and increases modifiability. The unique aspects of this program are the style of test building and of defining the links between questions.

The INPUTQUESTIONS procedure is devoted to textual input of main questions and subquestions with printed lists for verification. This is accomplished through standard iterative programming. The section labeled LOGIC: conducts a dialogue with the researcher detailing the question sequence to be followed. Response contingency is obtained by differentiating between what should follow a "true" response as opposed to a "false" response. The program expects question sequencing information in the form of two-digit numbers for questions and subquestions. This information is stored in the array variable QLOGIC. In response to the screen prompt "For main question 17, subquestion 4, what should follow a false subject response?" the researcher supplies a four-digit instruction. If the questionnaire logic requires an end to the current subquestions and an advance to subquestions for the next main question, then 1800 would be the appropriate instruction. If the situation called for an advance to Subquestion 5, then 1705 would be the correct instruction. This structure allows the researcher to direct subject questioning in great detail. Instructions must be supplied for all decision points. At the end of the dialogue, the variable QLOGIC contains a matrix of four-digit numbers that are in turn translatable into instructions. This process allows the program to store actual question text as simple linear sequences and yet provide a rapid and powerful branching logic. Those considering modification of the Flexible Tester should note that the variable QLOGIC is a three-dimensional array (main question index, subquestion index, false/true index) that contains the fourdigit sequencing instructions.

Because of the distinct possibility of becoming lost in the detailed links between questions, the Flexible Tester prints the contents of QLOGIC for all array cells. This provides a means whereby the branching and sequencing desire of the researcher may be verified.

Administration of questions and subquestions proceeds either as main questions followed by appropriate subquestions or with relevant subquestions inserted between main questions. The process ends with the instruction to proceed to the last main question plus one, which is the "End" message.

\section{Discussion}

The Flexible Tester is inflexible in several ways. Error trapping is limited, and instructions may be stated only in terms of where to go next. It is limited to specific machines, and modifications require access to TML BASIC. The program proceeds as directed without regard to the advisability of doing so, and it is unable to detect endless loops. It is perfectly possible for the researcher to create a series of instructions that repeat without end. It is highly recommended that the questioning process be diagrammed on paper in full detail, complete with text, false/true responses, and direction arrows. Only in this way can the user feel reasonably assured that endless loops have not been created. Clearly, there will be some undefined point at which a procedure has become too complex to manage.

The program allows 99 main questions and unlimited subquestions. The real limits are imposed by available memory, though TML BASIC does provide a means to increase the size of the memory available to the various programming functions. Exact limits will depend on the total memory available in each individual machine environment.

Future possibilities include translation to MS-DOS machines, provisions for the input of scoring keys for subquestions, facilities for the input of responses from copyrighted tests (thus not violating copyright), facilities for questionnaires that use ratings, and, lastly, facilities for linking different questionnaires for administration in a single session.

\section{Availability}

The Flexible Tester is available only for the Apple IGGS, operating under OS 1.0 through 3.1. The compiled version is available by sending a blank 3.5 -in. diskette and return information, plus postage, to the author. TML BASIC is available from most software dealers.

\section{REFERENCES}

Klieger, D. M. (1987). The Snake Anxiety Questionnaire as a measure of ophidophobia. Educational \& Psychological Measurement, 47, 449-459.

KLIEGER, D. M. (1989, April). The Snake Anxiety Questionnaire and 
false positives: Problems and solutions. Paper presented at the meeting of the Eastern Psychological Association, Boston.

Klorman, R., Weerts, T. C., Hastings, J. E., Melamed, B. G , - LANG, P. J. (1974). Psychometric description of some specific-fear questionnaires. Behavior Therapy, 5, 410-409

TML Systems INC. (1987). TML BASIC for the Apple I/GS [Computer program]. Jacksonville, FL: Author.
Weiss, D J (1985) Adapuve testıng by computers Journal of Consulting \& Clinical Psychology, 53, 774-789

WolPE, J., LANG, P J (1964). A fear survey schedule for use in behavior therapy Behavior Research \& Therapy, 2, 27-30

WOLPE, J., LANG, P J (1977). Manual for the fear survey sched. ule (rev. ed.). San Diego, CA: Educational \& Industrial Testing Service 